\title{
Radial velocities of planetary nebulae towards the Galactic bulge ${ }^{\star, \star \star}$
}

\author{
A.A. Zijlstra ${ }^{1}$, A. Acker ${ }^{2}$, and J.R. Walsh ${ }^{1}$ \\ 1 European Southern Observatory, Karl-Schwarzschild-strasse 2, D-85748 Garching bei München, Germany \\ ${ }^{2}$ Observatoire de Strasbourg, 11 Rue de L'Université, F-67000 Strasbourg, France
}

Received December 24, 1996; accepted January 3, 1997

\begin{abstract}
Accurate radial velocities for a sample of 71 planetary nebulae, mostly located in the direction of the Galactic Centre, are presented. Most of the nebulae discussed in this paper are likely members of the Galactic bulge, based on their radial velocity, longitude and derived distance. The data give a large improvement over existing catalogues, doubling the sample of bulge planetary nebulae with well-determined velocities and suggesting that kinematics of the Galactic Bulge can usefully be studied by planetary nebulae.
\end{abstract}

Key words: planetary nebulae - stars: kinematics Galaxy: center - Galaxy: kinematics and dynamics techniques: radial velocities

\section{Introduction}

Planetary nebulae are among the best velocity tracers of stellar populations and have as such been successfully used to trace the dynamics of extragalactic old stellar populations (e.g. Ciardullo et al. 1993). They are the most luminous objects among the old stellar population, are easy to find, with most of their flux coming out in a few bright emission lines, and yield accurate radial-velocity determinations using these narrow emission lines. In contrast to integrated stellar light, they are identifiable point probes of the gravitational potential field of the host galaxy.

In view of this, it is perhaps surprising that little use has been made of planetary nebulae for the study of the dynamics of the Milky Way. The high obscuration, especially towards the Galactic Centre, makes them difficult

\footnotetext{
Send offprint requests to: A. Zijlstra

* Based on observations obtained at the European Southern Observatory.

** Table 1 is also available in electronic form at the CDS vis anonymous ftp to cdsarc.u-strasbg.fr (130.79.128.5) or via http://cdsweb.u-strasbg.fr/Abstract.html
}

to detect, and their distances are unknown to significant factors. Schneider \& Terzian (1983) used PN for the outer Milky Way, deriving a rising rotation curve in the outer Galaxy. It seems possible that this result was dominated by Malmquist-like bias caused by uncertainties in the distances, which can cause systematic errors in the derived properties of a sample even if the scale itself has only nonsystematic effects. The same effect has caused the scale height of the PN population to be underestimated by a factor of 2 (Zijlstra \& Pottasch 1991). In the case of a flat Galactic rotation curve, either under- or overestimation of the distance to an individual object will lead to a positive velocity gradient.

The compilation of data in Schneider et al. (1982) is still the main source for velocities of Galactic planetary nebulae. They combined all measurements in the literature, correcting for systematic offsets between different sources. The data used are of varying accuracy. Recently, Kohoutek \& Pauls (1995) have presented well-determined radial velocities for 76 nebulae in the direction of the Galactic Centre. Spyromilio (1995) gives accurate velocities for a few non-bulge PN. In the present paper we present new data on 71 nebulae. The targets were selected on the basis of their Galactic longitude to be within 15 degrees of the Galactic Centre. Preference was given to objects with size and flux consistent with bulge membership, but very faint objects were not observed. Previous velocity determinations are available for many of the observed nebulae, but many of these are rather uncertain. Comparing the velocity as function of longitude and derived distance shows that most of the planetary nebulae discussed belong to the Galactic bulge.

\section{Observations and results}

The observations were carried out with the ESO $1.4 \mathrm{~m}$ CAT telescope in June and July 1993, and March 1994. In July, the short camera was used in the blue arm, with a resolution of 30000 , corresponding to $6 \mathrm{~km} \mathrm{~s}^{-1}$ and the [O III] $5007 \AA ̊$ Aine was observed. In June 1993 and March 
1994 the $\mathrm{H} \alpha$ line and neighbouring [N II] 6548, $6583 \AA$ lines were observed, using a resolution of 60000 . For $\mathrm{H} \alpha$ the higher resolution is partly offset by the larger thermal broadening. Integration times varied from a few to 30 minutes depending on the line flux. The faintest nebulae could not be observed because they were not visible on the finder due to the brightness of the Moon at both epochs. The slit had a width of $2^{\prime \prime}$ and a length of $20^{\prime \prime}$. No flux calibration was performed. The wavelength calibration was done with ThAr spectra, and also checked by observing well-known planetary nebulae as velocity standards.

The data reduction involved flat fielding, summation of the spectrum over the slit and wavelength calibration. The velocity scale was corrected for the Earth's motion to obtain heliocentric velocities. The radial velocity for the [O III] measurements was determined from a first-moment analysis over the line profile. For the measurements of $\mathrm{H} \alpha$ and the [NII] lines (plus in a few cases the nearby HeII $6560.1 \AA$ line) the central velocity between the two peaks was used instead. The uncertainty in the velocities is caused mainly by the method of averaging. The velocity profile is typically $10-20 \mathrm{~km} \mathrm{~s}^{-1}$ wide at half peak intensity. When it is symmetric, the central velocity is easy to define, but often one peak is somewhat stronger than the other or distortions can be caused by a bipolar outflow in combination with an off-centre slit. A different method to obtain velocities is to fit the wings of the line: Spyromilio (1995) finds that this works well, with the wings (defined as line-to-continuum ration less than $10 \%$ of the line peak) being more symmetric than the central part of the profile. This method cannot be easily used for our data because one wing is distorted at a low level due to charge-transfer deficiencies of the CCD. The total uncertainty in the velocities was derived by comparing with previous determinations of bright nebulae, for which agreement was found within $2 \mathrm{~km} \mathrm{~s}^{-1}$. Agreement to within $3 \mathrm{~km} \mathrm{~s}^{-1}$ is found between the $\mathrm{H} \alpha$ and [O III] measurements (reduced in different ways) for all 13 objects in common, with a mean (systematic) deviation of $1.5 \mathrm{~km} \mathrm{~s}^{-1}$. This indicates that within the adopted uncertainty, the velocities do not depend on the method of averaging. Higher resolution will not improve this accuracy, which is dominated by the internal motions of the nebulae affecting the moment calculation. In most cases the lines are resolved: detailed analysis of the profiles will be given in Gesicki et al. (1997).

The results are presented in Table 1 . The numbering convention is the one introduced by Acker et al. (1992) which has superseded the previous Perek \& Kohoutek (1967) scheme. Velocities are averaged over all available measurements.

Two objects in Table $1(004.8-22.7$ and $006.8-19.8)$ have velocities and positions in agreement with the newly discovered Sagittarius Dwarf galaxy and are probably members of this dwarf spheroidal orbiting our Galaxy (Zijlstra \& Walsh 1996) at a distance of $25 \mathrm{kpc}$. A third object in the same direction $(003.8-17.1, \mathrm{HB} 8)$ also has a large indicated distance but a much different velocity.

\section{Discussion}

For most of the nebulae in Table 1 older velocity measurements are given in Schneider et al. (1983). However, few of these are of comparable accuracy, where the uncertainty assigned by Schneider et al. appears in some cases to be an underestimate. A paper discussing the accuracies of the different data sets is in preparation (Durand et al. 1997). The problem is serious for the Galactic Bulge region where most of the velocities derived from two samples: Mayall (1964) and Minkowski (1957). Both are published only as private communications in the catalogue of Perek \& Kohoutek (1967) and may originate from a variety of separate measurements with different accuracies. The unknown uncertainties in these data sets are a significant problem for any attempt to study the dynamics of the inner Galaxy using PN velocities. There are only two other large sources of velocity data with comparable accuracy to the present survey: Kohoutek \& Pauls (1981), and Campbell \& Moore (1918), where the latter authors only study bright nebulae which consequently are mostly foreground objects. Values given by Campbell \& Moore agree to within $1 \mathrm{~km} \mathrm{~s}^{-1}$ : the only deviating object is $358.3-21.6$, where the velocity difference is $10 \mathrm{~km} \mathrm{~s}^{-1}$. This object has however a very complicated velocity field and the result is sensitive to the placement of the slit.

To illustrate that indeed the present nebulae are sampling the Galactic bulge, both distances and velocity distribution have to be shown to be consistent with expected values. The longitudinal distribution of the full sample of Galactic PN shows a sharp peak towards $l=0$ (Acker et al. 1992) indicating that there is a large Bulge population among the known PN. However, since the present objects were selected partly based on brightness, further confirmation is required.

The distances were estimated from the surfacebrightness-distance relation proposed by Van de Steene \& Zijlstra $(1994,1995)$ : for the sample they are listed in Table 1. (For the two PN which appear to be extragalactic the distance is instead that of the host galaxy. For neither PN can the distance scale be used for lack of a radio flux density and diameter, and they are not included in Fig. 1.) A very similar distance scale using optical flux determinations was independently derived by Schneider \& Buckley (1996); their scale may better fit the more compact nebulae which may be affected by optical depth effects at radio wavelengths. Use of the radio flux is in general preferred in regions of high extinction. For the present purpose both scales give the same result. The formula of Van de Steene \& Zijlstra is:

$\log D=4.36-0.35 \log S_{6 \mathrm{~cm}}-0.3 \log \theta_{\mathrm{r}}$ 
Table 1. Radial velocities of PN towards the Galactic bulge

\begin{tabular}{|c|c|c|c|c|}
\hline Source & Name & $\begin{array}{r}V_{\text {hel }} \\
\left(\mathrm{km} \mathrm{s}^{-1}\right)\end{array}$ & Note & $\begin{array}{l}\text { Adopted dist. } \\
(\mathrm{kpc})\end{array}$ \\
\hline $000.7-02.7$ & $\mathrm{M} 2-21$ & -164.7 & 1 & \\
\hline $000.9-04.8$ & M3-23 & -152.9 & 2,3 & 4.2 \\
\hline $001.5-06.7$ & SwSt1 & -16.1 & 1 & 4.7 \\
\hline $001.7-04.6$ & $\mathrm{H} 1-56$ & -116.3 & 1 & 10.4 \\
\hline $002.0-06.2$ & M2-33 & -126.0 & 1 & 8.0 \\
\hline $002.0-13.4$ & IC4776 & +16.8 & 1 & 3.5 \\
\hline $002.1-02.2$ & M3-20 & +24.1 & 1 & \\
\hline $002.2-02.7$ & $\mathrm{M} 2-23$ & +186.7 & 2 & 8.5 \\
\hline $002.6+08.1$ & $\mathrm{H} 1-11$ & +4.8 & 1,2 & 6.7 \\
\hline $002.6-03.4$ & M1-37 & +220.5 & 2 & 8.1 \\
\hline $002.7-04.8$ & M1-42 & -86.5 & 2 & $4.5^{b}$ \\
\hline $003.2-06.2$ & $\mathrm{M} 2-36$ & +73.0 & 1 & 6.4 \\
\hline $003.7-04.6$ & $\mathrm{M} 2-30$ & +155.8 & 1 & 7.7 \\
\hline $003.8-17.1$ & HB8 & -183.2 & 1 & 16.2 \\
\hline $003.9-02.3$ & M1-35 & +67.4 & 1,2 & $4.3^{b}$ \\
\hline $004.0-03.0$ & M2-29 & -112.0 & 1 & 9.3 \\
\hline $004.2-04.3$ & $\mathrm{H} 1-60$ & +24.8 & 1 & \\
\hline $004.6+06.0$ & $\mathrm{H} 1-24$ & +157.9 & 2 & $6.8^{a}$ \\
\hline $004.8-22.7$ & $\mathrm{He} 2-436$ & +132.9 & 1 & 25.0 \\
\hline $004.9+04.9$ & $\mathrm{M} 1-25$ & +14.1 & 1 & 4.8 \\
\hline $005.2+05.6$ & M3-12 & +17.1 & $1,2,3$ & 6.3 \\
\hline $005.8-06.1$ & NGC6620 & +69.8 & 1 & $6.1^{b}$ \\
\hline $006.0-03.6$ & $\mathrm{M} 2-31$ & +151.7 & 2 & 4.8 \\
\hline $006.1+08.3$ & $\mathrm{M} 1-20$ & +60.3 & 1,2 & 5.9 \\
\hline $006.4+02.0$ & M1-31 & +68.2 & 2 & $5.3^{a, b}$ \\
\hline $006.8+04.1$ & M3-15 & +97.9 & 1,2 & 3.9 \\
\hline $006.8-19.8$ & Wray16-423 & +133.1 & 1 & 25.0 \\
\hline $008.2+06.8$ & $\mathrm{He} 2-260$ & +21.5 & 2 & 11.5 \\
\hline $008.3-01.1$ & $\mathrm{M} 1-40$ & -34.2 & 1 & 2.8 \\
\hline $009.4-09.8$ & M3-32 & +58.7 & 1 & \\
\hline $009.6-10.6$ & M3-33 & +179.8 & 1 & 8.1 \\
\hline $010.7-06.4$ & IC4732 & -147.8 & 1 & 4.8 \\
\hline $014.0-05.5$ & $\mathrm{~V}-\mathrm{V} 3-5$ & -45.6 & 1 & \\
\hline $331.3+16.8$ & NGC5873 & -133.4 & 1 & 4.0 \\
\hline $334.8-07.4$ & SaSt2-12 & -63.0 & 2 & \\
\hline $342.1+27.5$ & $\mathrm{Me} 2-1$ & +44.4 & 2,3 & 4.8 \\
\hline $345.0-04.9$ & $\mathrm{CN} 1-3$ & -80.0 & 1 & \\
\hline $346.3-06.8$ & $\mathrm{FG}-2$ & +34.7 & 1 & \\
\hline $347.4+05.8$ & $\mathrm{H} 1-2$ & -105.0 & 1,2 & 7.1 \\
\hline $348.0-13.8$ & IC4699 & -122.7 & 1 & 5.5 \\
\hline $349.8+04.4$ & $\mathrm{M} 2-4$ & -207.9 & 1 & 6.7 \\
\hline $350.9+04.4$ & $\mathrm{H} 2-1$ & -18.5 & 1 & $5.1^{a}$ \\
\hline $351.1+04.8$ & M1-19 & -55.1 & 2 & $6.7^{a}$ \\
\hline $351.6-06.2$ & $\mathrm{H} 1-37$ & -23.2 & 1 & \\
\hline $351.9+09.0$ & $\mathrm{PC}-13$ & -75.2 & 1 & \\
\hline $352.1+05.1$ & $\mathrm{M} 2-8$ & +25.1 & 2 & 6.9 \\
\hline $352.9-07.5$ & $\mathrm{FG}-3$ & +0.5 & 1 & \\
\hline $353.3+06.3$ & $\mathrm{M} 2-6$ & -127.4 & 1,2 & $8.4^{a}$ \\
\hline $353.5-04.9$ & $\mathrm{H} 1-36$ & -118.8 & $1,2,3$ & \\
\hline $355.1-02.9$ & $\mathrm{H} 1-31$ & +47.0 & 1 & 11.8 \\
\hline $355.1-06.9$ & M3-21 & -66.1 & 1 & 5.3 \\
\hline $355.4-02.4$ & M3-14 & -82.3 & 1 & $8.1^{a}$ \\
\hline $355.7-03.5$ & $\mathrm{H} 1-35$ & +123.3 & 1,2 & $5.1^{a}$ \\
\hline $355.9+03.6$ & $\mathrm{H} 1-9$ & -157.6 & 2 & $9.4^{a}$ \\
\hline
\end{tabular}

Table 1. continued

\begin{tabular}{llrcl}
\hline Source & Name & $\begin{array}{r}V_{\text {hel }} \\
\left(\mathrm{km} \mathrm{s}^{-1}\right)\end{array}$ & Note & $\begin{array}{l}\text { Adopted distance } \\
(\mathrm{kpc})\end{array}$ \\
\hline $356.2-04.4$ & CN2-1 & -169.1 & $1,2,3$ & $6.9^{a}$ \\
$356.5-02.3$ & M1-27 & -49.7 & 2 & 3.5 \\
$356.9+04.5$ & M2-11 & +84.5 & 1 & $7.1^{a}$ \\
$357.1+03.6$ & M3-7 & -189.7 & 1 & \\
$357.2+07.4$ & M4-3 & +132.1 & 1,2 & 8.1 \\
$357.4-03.2$ & M2-16 & +89.6 & 2,3 & $6.9^{a}$ \\
$357.4-03.5$ & M2-18 & -21.3 & 2 & 9.2 \\
$358.2+03.6$ & M3-10 & -131.4 & $1,2,3$ \\
$358.3-21.6$ & IC1297 & +9.1 & 1 & \\
$358.5-04.2$ & H1-46 & -44.3 & 1,2 & 7.1 \\
$358.7-05.2$ & H1-50 & +70.4 & 1 & 7.6 \\
$359.1-02.3$ & M3-16 & +72.0 & 2 & 5.9 \\
$359.1-01.7$ & M1-29 & -41.3 & $1,2,3$ & 3.2 \\
$359.3-00.9$ & HB5 & -22.9 & 1 & 1.3 \\
$359.7-02.6$ & H1-40 & +63.8 & 1 & 7.8 \\
$359.8-07.2$ & M2-32 & -53.6 & 1 & \\
$359.9-04.5$ & M2-27 & +145.0 & 1,2 & \\
\hline
\end{tabular}

Notes: 1 [O III] velocity; $2 \mathrm{H} \alpha$, [N II]; 3 HeII ${ }^{a}$ Using the angular size of Bedding \& Zijlstra (1994).

${ }^{b}$ Using radio flux density of Pottasch \& Zijlstra (1994).

where $D$ is the distance in pc, $S_{6} \mathrm{~cm}$ the 6 -cm radio flux in mJy and $\theta_{\mathrm{r}}$ the angular radius in arcseconds. The use of radio data overcomes the problem of extinction correction. From a calibration to nearby nebulae and likely bulge nebulae, an average uncertainty of $30 \%$ in the calculated distance was derived for this scale, which compares favourably with other distance scales, but is still a large uncertainty which could introduce significant bias in any attempt to derive a rotation curve. The radio flux density and angular radius as listed in Acker et al. (1992) were used, except where superseded by more recent data. Single-dish radio flux measurements were not used. The distances are in most cases between 4 and $9 \mathrm{kpc}$. This indicates the presence of a Bulge population. However, the distribution is skewed towards distances less than the Bulge, with a median distance of about $6 \mathrm{kpc}$. This skewness is absent in the distance scale used (see Van de Steene \& Zijlstra 1995) and indicates the presence of a bias. This does not necessarily imply a large fraction of non-bulge objects, since the Bulge has a large depth and the brightness selection could have favoured objects within the near part of the Bulge.

The velocity distribution as function of longitude and derived distance is shown in Fig. 1. In the top panel are shown all objects with an estimated distance $>4 \mathrm{kpc}$ from the Sun. Indicated are curves representing velocities expected from pure circular rotation with $V(\theta)=200 \mathrm{~km} / \mathrm{s}$, for distances from the Sun of 6 and $7.5 \mathrm{kpc}$. The distance to the Galactic Centre is taken as $8 \mathrm{kpc}$. The velocity 


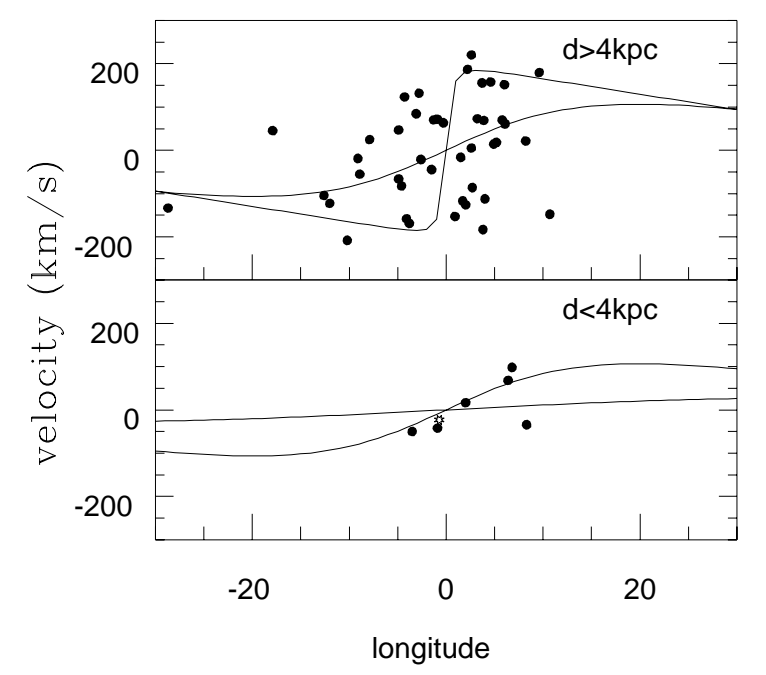

Fig. 1. Heliocentric velocity versus Galactic longitude of Galactic planetary nebulae. The top panel shows all PN at distances $>4 \mathrm{kpc}$ from the Sun which are possible Bulge members. The bottom panel shows PN nearer the Sun. The continuous lines indicate expected radial velocities based on Galactic (disk) rotation at distances of 6 . and $7.9 \mathrm{kpc}$ (top panel), and 2 and $6 \mathrm{kpc}$ (bottom panel). In the bottom panel, the open symbol indicates the single object in the sample at a distance of less than $2 \mathrm{kpc}$

distribution is consistent with the objects being near the Galactic Centre with significant dispersion of $114 \mathrm{~km} \mathrm{~s}^{-1}$. The distribution of the data points does not agree with disk rotation and instead is dominated by the velocity dispersion. In contrast, $\mathrm{PN}$ with estimated distance of less than $4 \mathrm{kpc}$, shown in the bottom panel of Fig. 1, show a velocity-longitude distribution much more consistent with the general Galactic rotation. The velocity dispersion of all $\mathrm{PN}$ in the sample with distance of less than $4 \mathrm{kpc}$ is $46 \mathrm{~km} \mathrm{~s}^{-1}$. Note that we cannot rule out that some of the nebulae in Fig. 1a are dynamically part of the inner disk rather than the bulge. However, the derived velocity dispersion agrees well with other determinations for the Bulge (e.g. Minniti 1996), which indicates that sample confusion is relatively small.

The inner Galaxy contains, in addition to the Bulge and inner disk, a stellar population belonging to the metalpoor halo (Minniti 1996). It is possible that some of the PN of Table 1 belong to this population. The abundance distribution of Bulge nebulae shows a tail of objects with $[\mathrm{O} / \mathrm{H}] \approx-1$ (Ratag et al. 1992). These are expected to have a $[\mathrm{Fe} / \mathrm{H}]<-1$, based on models of chemical evolution (e.g. Matteucci \& Brocato 1990), and to fall in the range of metallicities found for the halo component in the Bulge region (Minniti 1996). However, it is known that the birth rate for $\mathrm{PN}$ within the halo population is up to a factor of 10 lower than in more metal-rich (younger?) populations (e.g. Zijlstra \& Walsh 1996; Jacoby 1996).
Thus, even in the presence of a significant halo population within the Bulge region, few PN would be expected to belong to this.

We conclude that the nebulae in Table 1 with estimated distance $>4 \mathrm{kpc}$ are mostly members of the Bulge. However, it is likely that preferentially the near side of the Bulge was sampled. To obtain a more complete sample for the Bulge, it will be necessary to include fainter nebulae. This problem will be studied further in the forthcoming paper by Durand et al. (1997).

\section{Conclusions}

In conclusion, the velocities presented here give a significant improvement over older data for planetary nebulae in the Galactic bulge. The majority of these nebulae in the direction of the Bulge have estimated distances and velocities consistent with bulge membership.

Acknowledgements. We are grateful to Bjorn Stenholm who participated in some of the observations, and to Dante Minniti who gave helpful comments.

\section{References}

Acker A., Ochsenbein F., Stenholm B., Tylenda R., Marcout J., Schon C., 1992, Strasbourg-ESO Catalogue of Galactic Planetary Nebulae

Bedding T.R., Zijlstra A.A., 1994, A\&A 283, 955

Campbell W.W., Moore J.H., 1918, Publ. Lick Obs. 13, 75

Ciardullo R, Jacoby G.H., DeJonghe, H.B., 1993, ApJ 414, 454

Durand S. Acker A., Zijlstra A.A., et al., 1997 (submitted to A\&A)

Jacoby G.H., 1996, in: IAU Symposium 180, Planetary Nebulae Gesicki K., Zijlstra A.A., Acker A., 1997 (in preparation)

Perek L., Kohoutek L., 1967, catalogue of galactic planetary nebulae. Czechoslovak Institute of Science, Prague

Kohoutek L., Pauls R., 1995, A\&AS 111, 493

Matteucci F., Brocato E., 1990, ApJ 365, 539

Mayall N.U., 1964, in: Perek \& Kohoutek (1967) (private communication)

Minkowski R., 1957, in: Perek \& Kohoutek (1967) (private communication)

Minniti D., 1996, ApJ 459, 579

Pottasch S.R., Zijlstra A.A., 1994, A\&A 289, 261

Ratag M..A., Pottasch S.R., Dennefeld M., Menzies J.W., 1992, A\&A 255, 255

Schneider S.E., Terzian Y. Purgathofer A., Perinotto M., 1983, ApJS 52, 399

Schneider S.E, Buckley D., 1996, ApJ 459, 606

Schneider S.E., Terzian Y., 1983, ApJ 274, L64

Spyromilio J., 1995, MNRAS 277, L59

Van de Steene G.C., Zijlstra A.A., 1994, A\&AS 108, 485

Van de Steene G.C., Zijlstra A.A., 1995, A\&A 293, 541

Zijlstra A.A., Walsh J.R., 1996, A\&A (Letters) 312, L21 